\title{
Design and Realization of New Generation Campus Smart Card System Based on the Mobile Communication
}

\author{
Qijie Sheng, Xin Tong, Xiangyang Song, Zhihao Zhang \& Jun Xu \\ Department of Finance, Northwestern Polytechnical University, Xi'an 710072, China \\ E-mail:shengqijie@nwpu.edu.cn
}

\begin{abstract}
The whole framework of the new generation campus smart card system based on the mobile communication is designed in this article, and it realizes two technical breakthroughs when it could remain compatible with the existing campus smart card system platform. The first technology is that the design could use the wireless POS based on GPRS network to substitute the wire POS to implement the online identify certification and the consumption payment function at any place, and the second technology is to use the mobile banking and RFID-SIM card composite technology to realize three cards (including the campus smart card, the mobile telephone card, and the bank card) in one card, and use the mobile telephone to substitute the campus smart card campus smart card transfer machine to realize the wireless transfer and the online inquiry function. This application mode integrating the RF card and the mobile communication technology will be the new direction for the development of the campus smart card system, with wide application foreground.
\end{abstract}

Keywords: Mobile communication, RFID-SIM card, Campus smart card

\section{Introduction}

In 1990, Professor Kenneth Green in US Claremont College initiated and took charge a large scientific research project, i.e. "the Campus Computing Project", and first put forward the concept of "digital campus". At Jan 31 of 1998, the former vice president of US, Albert Gore delivered a speech of "the Digital Earth: Understanding Our Planet in the $21^{\text {st }}$ Century" in the California Center of US, and first put forward the concept of "digital earth", and derived many concepts such as "digital city" and "digital campus" (Blagwest Sendorfer, 1998). Through almost twenty years' exploration and research, foreign and domestic colleges have more deeply cognized the definition and connotation of the education informationization, i.e. the education informationization is the process taking information as a kind of basic element of the education system, and widely utilizing the information technology in various domains of education, and promoting the modernization of education. Moreover, the digital campus construction is to use many advanced information technical measures such as network, computer, and multimedia to implement the complete informationization of the teaching, scientific research, management and service in the campus, and form a whole network virtual space, and realize the enhancement of management efficiency and quality in the network campus by the virtual space $(\mathrm{Xu}, 2005)$.

The campus smart card system is the basic project of the digital campus. It has two functions such as identity certification and E-Wallet. It can offer large convenience for college staffs and students during their work, study, and daily life. At present, the current universal campus smart cards in the world adopt the Mifare one chip. But in 2008, Germany researcher Henryk Plotz and UVA (University of Virginia) PHD student of computer science Karsten Nobl successfully broke the security algorithm of the Mifare classic chip of NXP semiconductor (http://www.yqsh.com/news-company.aspx?articleid=320). Noncontact IC cards of Mifare series are the classic IC card products of the Holland Philips, and they mainly include four types such as Mifare one S50 (1K bytes), S70 (4K bytes) which have widely used in the entrance guard, campus and public traffic, simplified Mifare Light and updated Mifare Pro. In these chips, except for Mifare Pro, they all belong to the logical encryption card without independent CPU and operation system in the interior, and they all completely depend on the interior hardware logic circuit to realize security certification and protection (http://yxk.cn.yahoo.com/articles/20090903/n6c.html). Therefore, the application and spread of the noncontact CPU card will be the certain tendency.

In addition, the current campus smart card systems widely use many wire transmission modes such as RS485 communication cable, unshielded twisted pair, and optical fiber. This wire transmission mode could not fulfill the campus information service demands at anytime and anywhere, so the campus smart card system based on wireless transmission will be the technical direction in the further development. The new generation campus smart card system based on mobile communication which is designed in this article adopts the double-interface intelligent card (RFID card and SIM card) technology, and utilizes the GPRS network to realize the security information service at anytime and anywhere, and achieves the new ambit of "all-in-one and all-well- 
communication".

\section{Design of the whole framework of the new generation campus smart card system}

The whole frame of the campus smart card system based on mobile communication is seen in Figure 1, and it includes four parts.

(1) Unified data storage and data processing center. The data storage and data processing center is used to store and process the background data of the campus smart card system and various subsystems of management and application, and various consumption and identification terminals would utilize the technology based on RFID-SIM card to transfer data by the mobile communication service network.

(2) Meeting with the bank-end server and the front-end processor. This interface mainly is used to safely transfer the information such as the bank transfer, the balance inquiry of bank card, the tuition-payment agency, the response information return to the bank-end, and the daily account checking information, which are all initiated by the campus smart card platform.

(3) Meeting with the mobile communication server network. This interface is mainly used to transfer the consumption data, the inquiry data, the operation data, the customized operation information, the mobile micropayment information, and the daily account checking information of the campus smart card.

(4) Various subsystems of management and application. Various management subsystems could offer customized management platform for the school management institution, and the application subsystem mainly offers the application system platform for various commercial tenants and concrete service terminal, corresponding system front-end processor, and corresponding system sub-server for users.

\section{Key technologies of the mobile communication campus smart card system}

The new generation campus smart card system based on mobile communication could fully utilize the advantages of the GPRS network, such as permanent online, wide coverage, high quality communication, and safe data communication, and realize following technical breakthroughs based on the exiting campus smart card system platform. (1) The design could use the wireless POS based on GPRS network to substitute the wire POS to implement the online identify certification and the consumption payment function at any place. (2) The system uses the mobile bank and RFID-SIM card composite technology to realize three cards (including the campus smart card, the mobile telephone card, and the bank card) in one card, and uses the mobile telephone substitute the campus smart card transfer machine to realize the wireless transfer and the online inquiry function.

\subsection{Wireless POS technology based on the GPRS network}

\subsubsection{Design principle of wireless POS system}

The GPRS network transmission technology has many characters such as wide coverage, reliable communication, and stable transmission. As the communication carrier of the mobile POS trade of the campus smart card, it could realize the real-time trade and certification of the campus smart card without the limitations from place, environment, and lines. The technical characters of the GPRS network decide that the increase of wireless mobile POS and the development of the application will not influence the speed and successful rate of the trade of the campus smart card. The design principal of this system can be described as that (1) the GPRS DTU with the function of GPRS wireless data transmission incepts the punch data of the smart card POS machine by the interface RS485. (2) GPRS DTU communicates with the GPRS base station by the wireless mode, and transmits the data package sent by the POS machine from the base station to the node of SGSN which supports the communication with the node GGSN which correspondingly processes the coupling data and transmits it to the router on the campus smart card platform, and to the host computer of the campus smart card through the firewall. (3) the data finally return by the former path through the data package processed by the background server of the campus smart card, to the POS of the smart card system by GPRS DTU, so the data exchange between the campus smart card system with the GPRS network is completed, and the wireless data transmission of the POS system is realized. At the same time, the L2TP is used to establish the special enterprise virtual network on the GPRS network and ensure the specialty and durative of the network connection. The work sketch is seen in Figure 2.

\subsubsection{Wireless POS hardware design}

(1) Wireless POS terminal. This system adopts the 51 series SCM model as the hardware principle support of the terminal POS which is mainly composed by CPU (SM5964), FLASH data memory AM29F040B/39SF020 reset chip IMP810, 485 communication chip SN75174 and MAX487, RF card reader-writer, customer side LED display, operation side LED display, linear power supply, master keyboard, and password keyboard port, and its 
work principle is seen in Figure 3.

(2) GPRS DTU. GPRS DTU and GPRS network charge for the communication transfer of data in the system, and the GPRS network is based on the IP protocol, so the GPRS DTU in the system adopts the interior TCP/IP protocol stack, and it has the function of Socket communication, and offers the transparent data transfer channel. After the computer starts each time, GPRS DTU would dial automatically, meet with GPRS network and dynamically gain one IP address which is distributed by the interior network address pool of GPRS backbone, and when GPRS DTU transmits the connection request to the background server of the campus smart card system through GPRS network, and the background server of the campus smart card system returns the answer and the connection route is established. To ensure the free-flowing of the chain route, the "heartthrob" data package is transmitted each time in 30 seconds to maintain the route between the GPRS DTU terminal and the background server of the campus smart card system and ensure the timely communication between the terminal with the server. The "heartthrob" data package includes the chain keeping-link function information and the terminal ID information.

\subsubsection{Wireless POS software design}

(1) GPRS remote communication system software design. The system bottom software of the GPRS remote communication system in the system adopts the modularized design method, and the language is the standard C language, and various functional modules are realized by the subprograms, and the main program transfers the subprograms to realize various functions designed by the system. The GPRS communication terminal software logical flow is seen in Figure 4.

The software logical flow is explained as follows.

A. The system completes the GPRS initialization, meets GPRS network and dynamically gets an IP address.

B. Validating whether the system allows the wireless mobile POS to upload data. If it allows, the connection is established. Otherwise, the request of uploading will be delayed

C. Dialing network, and consulting with PPP, and establish the link of TCP/IP.

D. Wireless mobile POS exchanges data with the background server of the campus smart card through TCP or UDP protocol in the GPRS network.

E. When wireless mobile POS receives the control order, it will firstly validate the verified of the order, and then execute corresponding operation according to the order.

(2) Wireless mobile POS system operation flow design. The wireless mobile POS system is the core of the software operation system unit, and its work flow chart is in Figure 5.

\subsection{Three- in- one: campus smart card, mobile telephone card, and bank card}

\subsubsection{RFID-SIM card technology}

The RFID-SIM card not only has the same mobile communication function with common SIM card, but also could implement the wireless short-range communication by the antenna and the card reader attach on it, thereby it could extend to the non-typical domain including the function of on-site payment of mobile telephone and the function of identity certification. RFID-SIM card supports the contact work interface and the noncontact work interface, and the contact interface controls the application of SIM card, and completes the normal function of the mobile telephone card, such as call and message. At the same time, the noncontact interface could realize many applications such as noncontact consumption, entrance guard, and work attendance. Because supporting relative criterions (OTA and WIB) of air loading, the users of RFID-SIM card could update the application programs or the account deposits by the mode of air loading, and become the mobile telephone as the intelligent E-wallet which could be deposited at any time. The size of this card is completely consistent with traditional standard SIM card, and based on the frequency range of $2.4 \mathrm{G}$, it is a mobile telephone intelligent card which could realize the wireless communication function of short, normal, and long distances, and the communication distance of card punch is $10-500 \mathrm{CM}$, and it supports $100 \mathrm{M}$ from the single direction (data broadcast). RFID-SIM card has COS (micro operation system), and it has the high-level security management measures, which is different with the logic encrypt card, and it could read and write the card information by the mobile phone instantaneously (http://baike.baidu.com/view/2884446.htm?fr=ala0_1).

\subsubsection{Mobile bank technology}

The mobile telephone bank is composed by the mobile telephone, the GSM message center, and the bank system. In the operation process of the mobile bank, the user sends the order by the menu of SIM card, and the SIM card 
generates the message with regulated format and encrypt the message according to user's order, and transmits it to corresponding bank system according to corresponding application or address, the bank pretreats the message, and transforms the order as the system format of the host computer, and the host computer of the bank treats user's request, and returns the result to the bank interface system, and the interface system transforms the treated result as the message format, and the message center sends the message to users. The mobile bank mainly adopts STK, SMS, BREW, WAP and other implementation modes. This article adopts SMS (Short Message Service), i.e. utilizing the short message to deal with the bank operation, and the main advantage of this mode is to meet with users easily. The short message service has been the most usual communication mode among mobile users, and the mobile bank has the SMS function. Account information such as the amount of salary, the remittance, and the deposit all can be informed to users by the SMS mode. Taking ICBC as the example, its mobile bank service could be applied in various mobile telephones from various manufactures and types, as long as the user edit and send the short message with appointed format, the mobile bank clients could acquire the round-the-clock service with 7 days, they can freely inquire their accounts, and the transfer and remittance capitals could be delivered instantly, and the mobile bank could also donate, and pay telephone and mobile telephone charges (http://baike.baidu.com/view/427365.htm).

\subsubsection{Wireless transfer and online inquiry technology}

(1) The wireless transfer operation of the campus smart card. To realize the demand targets such as quick and high efficiency E-consumption, automatic identity certification, restriction without place and time, the interior of the RFID-SIM card designed in this article has many E-wallets for the security demand of the campus smart card account and the micropayment account, and the accounts are relatively independent, and it includes many parts such as the E-wallet of the campus smart card, the E-wallet of the mobile telephone, and the E-wallet of other commercial tenants (Sheng, 2010). The wireless transfer operation utilizes the SMS technology to establish the SMS mobile telephone bank communication mechanism with the signatory bank through the mobile communication service network, and offers the wireless recharge service for the E-wallet of the campus smart card, and really realize three in one (the campus smart card, the mobile telephone SIM card, and the bank card). The wireless transfer operation flow of the campus smart card is seen in Figure 6.

A. Cardholder initiates the mobile telephone transfer request through the interior function menu of the mobile telephone which transforms RFID-SIM account, bank account, transfer amount, campus smart card consumption password, and other user's orders into regulated format massages and encrypts these messages, and transmits these encrypted messages to the GSM network.

B. The GSM system receipts messages and pre-treat them, and transforms the orders into the format according with the host computer system of the smart card, and transmits the operation request to the transfer front-end processor, and the transfer front-end processor first validates whether the trade is legal by the identity front-end processor and the comprehensive operation front-end processor, and if the campus smart card identity certification and the E-wallet password validity are passed, the transfer front-end processor will initiates the trade request to the bank front-end server.

C. The bank front-end server realizes the transfer of the bank card account capitals through the bank interior financial system, and returns the result to the transfer front-end server.

D. After the transfer front-end processor receives the information returned by the bank front-end server, it submit the information to the integrated operation front-end processor, and the database server updates the account balance of the campus smart card, and returns the result after successfully updating.

E. The transfer front-end processor submit the result to the GSM system, and the GSM system encapsulates the processing result information by the format of short message and transmits it to the cardholder's RFID-SIM mobile telephone, and the cardholder gets the trade result.

F. The reversal: if the transfer trade initiated to the bank exceeds the time, the system will automatically transmit the reverse trade of the transfer request to the bank, which could effectively reduce the inconsistency between the account recharge of the campus smart card with the account deduction of the bank card.

G. The Reconciliation: the system would check the accounts of the local trades and the trades of the bank each night, and if two parties differ, the system will enter into the manual processing mechanism. And if the bank account exceeds the local account, the system will increase the capitals to the cardholder's campus smart card by the form of subsidy.

(2) The mobile online inquiry operation. The mobile online inquiry operation includes the inquiry operations of various application subsystems on the campus smart card system platform, and it generates the SMS order by the 
interior function menu of the RFID-SIM card of the mobile to initiate the inquiry request, and the campus smart card platform charges for the implementation of the inquiry, and the inquiry result information is returned to the cardholder's RFID-SIM card telephone by the mode of short message, and the online inquiry function is completed. The concrete inquiry information mainly includes following sorts.

A. Inquiry of RFID-SIM campus smart card information: inquiry of cardholder's basic information, daily sequential inquiry, historical sequential inquiry, balance inquiry of the campus smart card, balance inquiry of the bank card, inquiry of commercial tenants' information, and inquiry of leader's info.

B. Inquiry of charge and registration information: inquiry of arrear information, inquiry of charge details, and inquiry of registration information.

C. Inquiry of charge agency information: inquiry of network charge information, inquiry of water and electric charge information, inquiry of telephone charge balance, and inquiry of examination charge.

According to the different required information query, the system initiates the inquiry operation by the transfer front-end processor, and searches the information demands of users' in different operation systems. The information inquiry operation flow is seen in Figure 7.

\section{Conclusions}

The whole frame of the campus smart card system based on mobile communication is designed in this article, and further discussed the key problems which need to be resolved. Combining with the function of mobile bank, the RFID-SIM card could be used to solve the problem of three cards (the campus smart card, the mobile telephone card, and the bank card) in one, and realize the transfer and inquiry function by taking the mobile as the mobile terminal. The design could use the wireless POS based on GPRS network to substitute the wire POS to implement the online identify certification and the consumption payment function at any place. With the continual development of the information technology, and the continually updating of many wireless networks such as GPRS and 3G, the application mode combining the campus smart card with the mobile communication technology will have wider application foreground.

\section{References}

Blagovest Hristov Sendov. (1997). Toward global wisdom in the era of digitalization and communication, Prospects, 27, n. 3, 415-426.

Sheng, Qijie \& Tong, Xin. (2010). Design of the Multi-media Self-service System Based on the Campus Smart Card Platform. China Education Info. No.2.

Xu, Xin \& Su, Xinning. (2005). Building a New Generation Digital Campus of University. New Technology of Library and Information Service. No.1. 


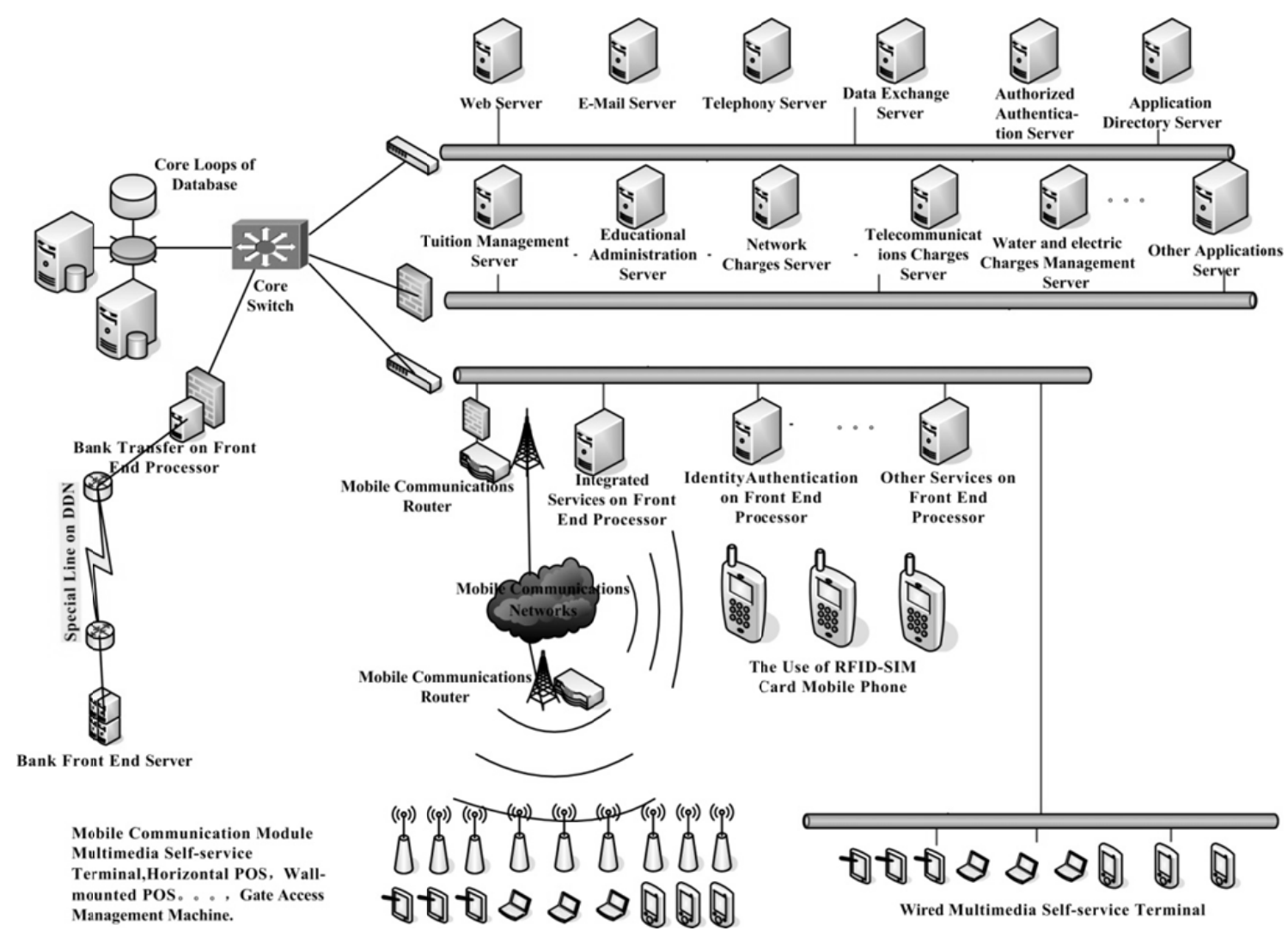

Figure 1. The Whole Framework of the Campus Smart Card System Based on the Mobile Communication

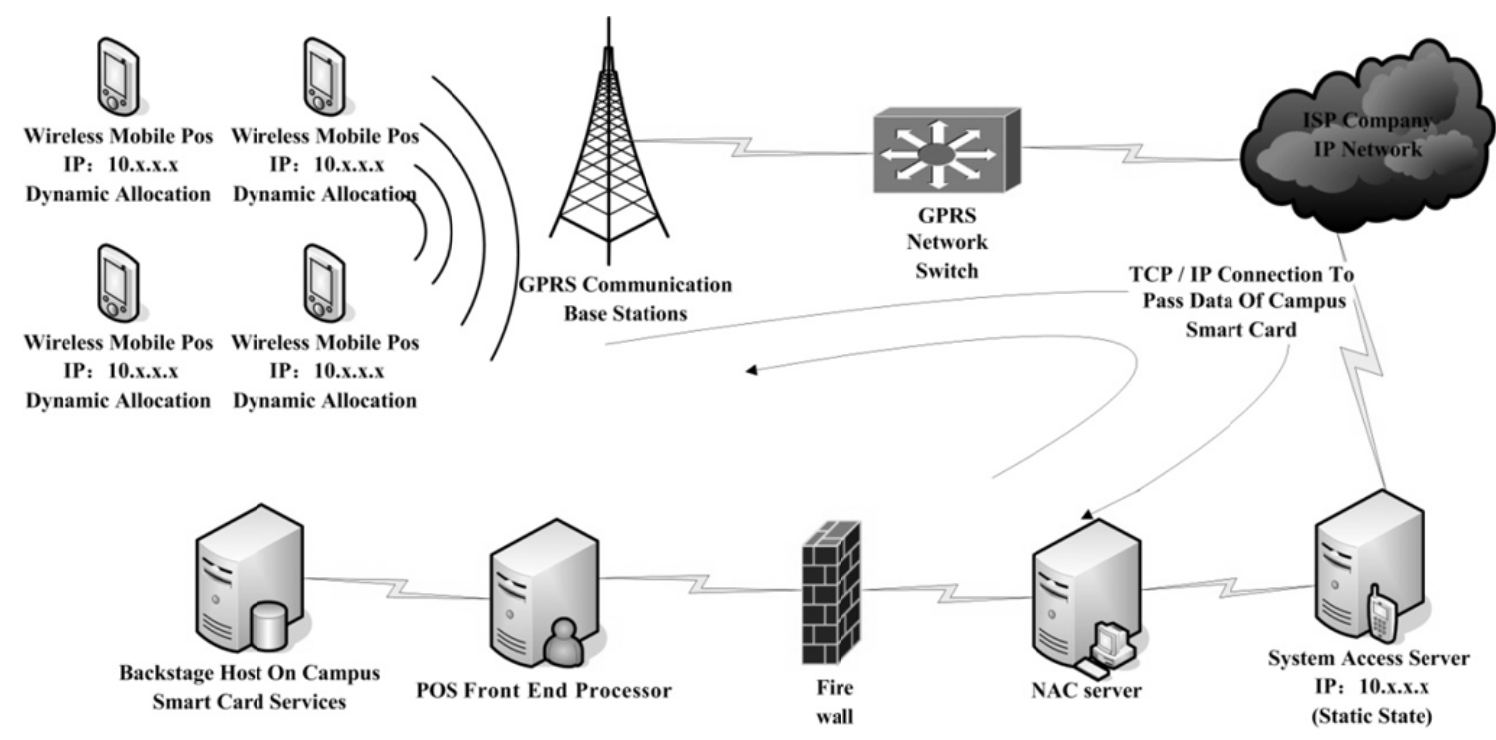

Figure 2. Communication Principle of the Campus Smart Card System Based on the Mobile Communication 


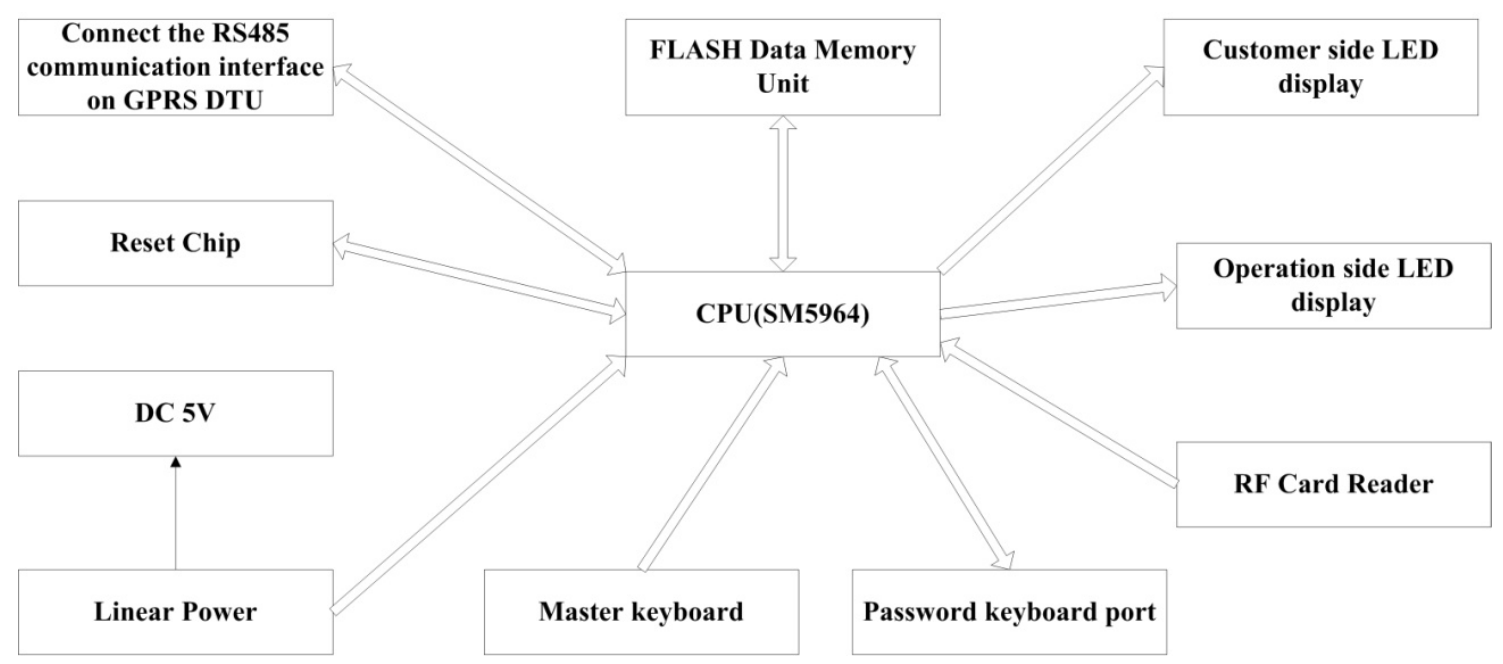

Figure 3. Hardware Principle of the Campus Smart Card Terminal POS Equipment

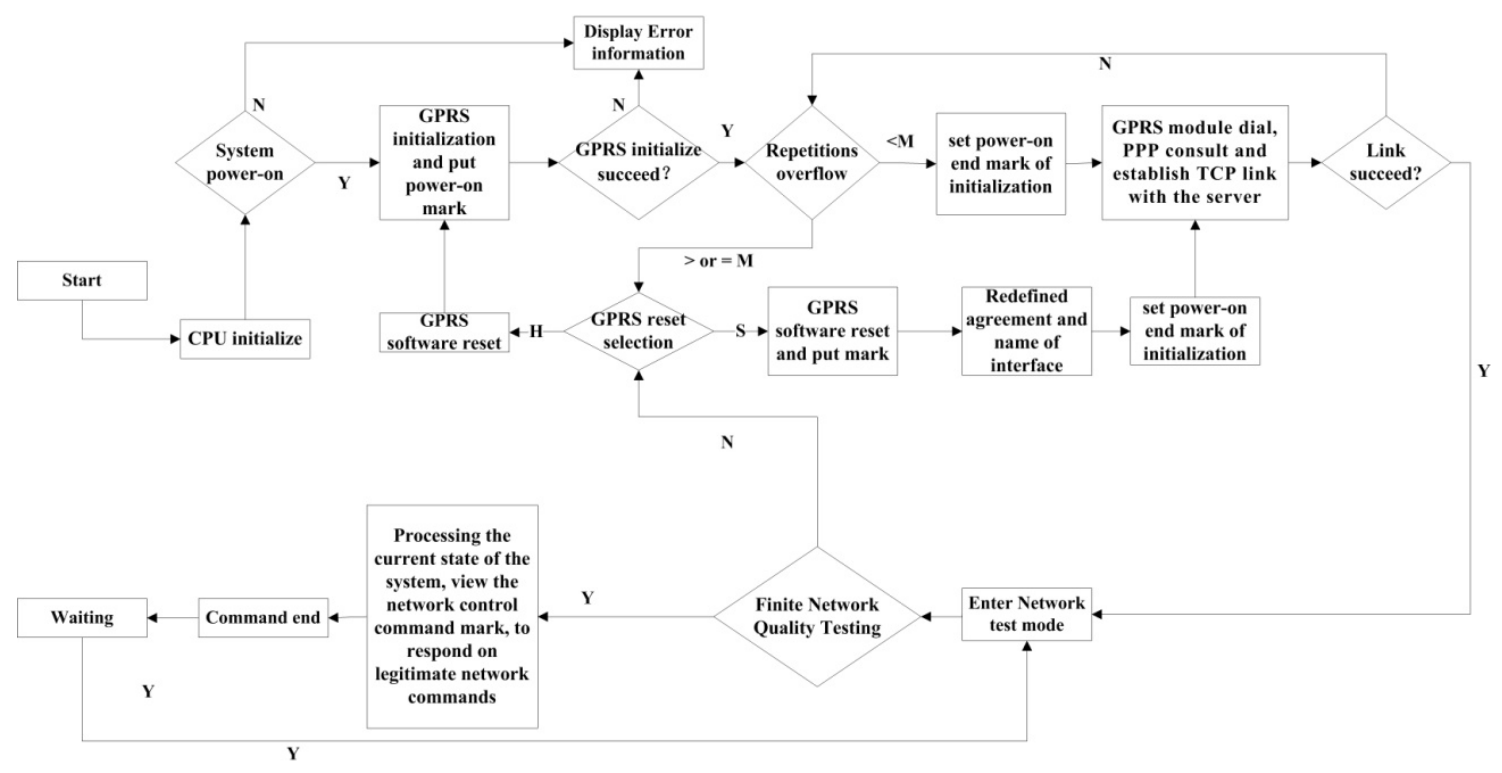

Figure 4. Main Program Logic Flow of the GPRS Remote Communication Terminal Bottom Software 


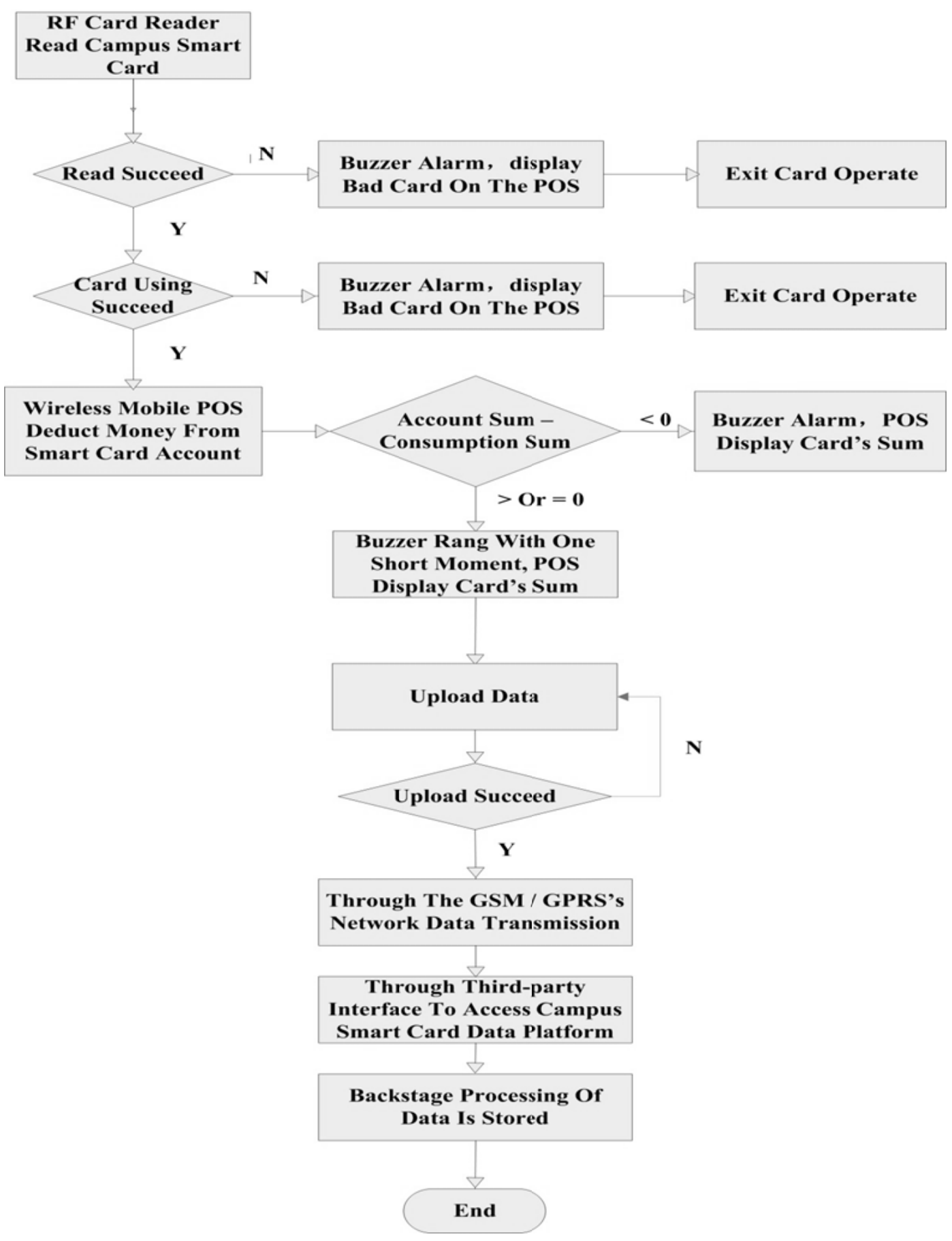

Figure 5. Operation Flow of the Wireless POS system 


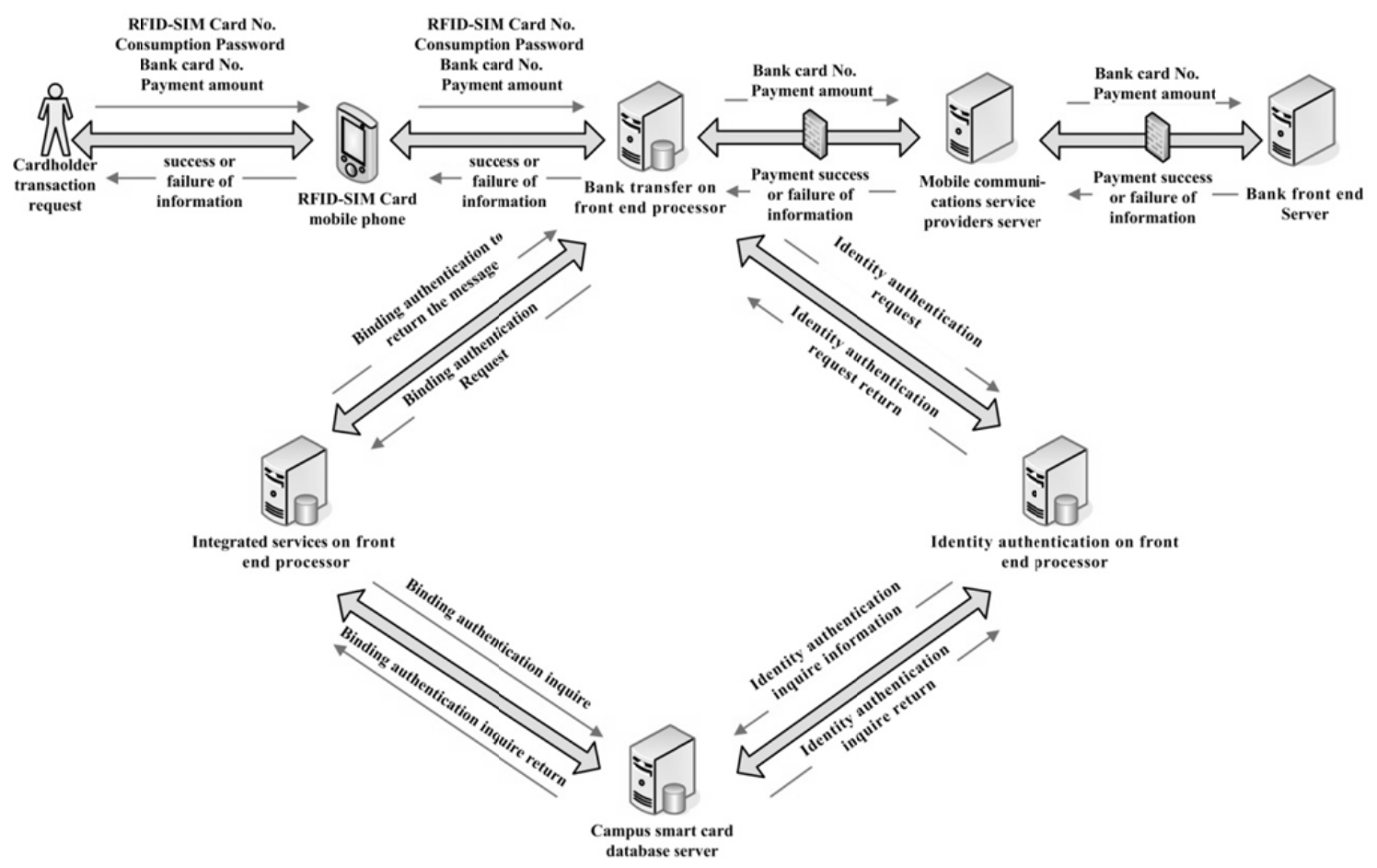

Figure 6. Operation Flow of the Campus smart card Wireless transfer

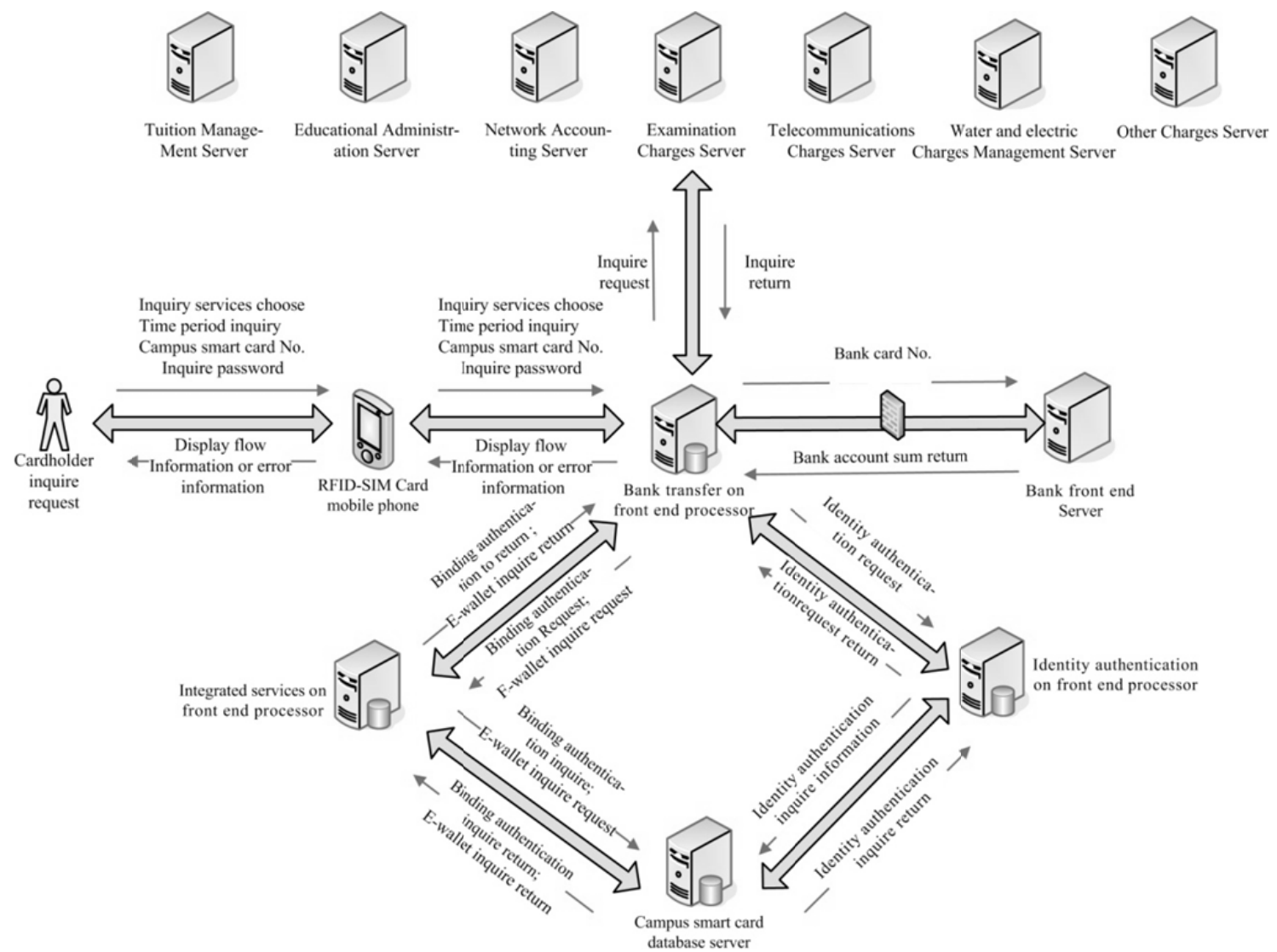

Figure 7. Operation Flow of the Mobile Telephone Online Inquiry 